\title{
PREDICTING FACTORS LEADING TO CARDIAC ARREST IN SOLID ORGAN TRANSPLANT RECIPIENTS: A RETROSPECTIVE ANALYSIS OF CODE BLUE PATIENTS.
}

\author{
Subhash P Acharya, ${ }^{1}$ Laura Hawryluck, ${ }^{2}$ Stuart McCluskey, ${ }^{3}$ Andrew Steel ${ }^{4}$ \\ *Correspondence to: Dr. Subhash Prasad Acharya, Department of Anesthesiology, Institute of Medicine, Tribhuvan University. \\ Email:drsuvash@gmail.com
}

\begin{abstract}
Background: Cardio Pulmonary (Code Blue) arrests in solid organ transplant recipients are particularly distressing events in view of the tremendous investment by organ donors, families, and by the healthcare system.

Methods: After ethical approval, all code blue events occurring in solid organ transplant patients were identified from Critical Care Response Team (CCRT) database and the code blue resuscitation records from 2007-2011. All patients who sustained cardio-respiratory arrests were included. Resuscitation records were also explored to identify quality, duration and immediate event and outcome.

Results: Over the five-year period, there were 63 code blue calls in solid organ transplant patients out of which only 27 $(n=27)$ were actual code blue arrest requiring resuscitation. The frequency was highest in liver transplant (10), followed by lungs (8), kidney (5), double organ (kidney + liver/lung $=2$ ), and heart (1), and one patient with Liver transplantation had arrested twice. Seventeen (62.96\%) of these patients were in the ward while $10(37.04 \%)$ were in the step-down unit (SDU) when the arrest occurred. Most of them $(16,59.25 \%)$ were attended by a nurse within 30 -mins prior to the code. Factors associated in these code blue patients were documented source of infection $(\mathbf{1 1}, \mathbf{4 0 . 7 4 \% )}$, blood transfusion $(7,23.9 \%)$, surgical event as cause of arrest $(6,22.22 \%)$, procedures within 24 hours $(6,22.22 \%)$ and dialysis within 24 hours $(5,18.51 \%)$. It was also found that serum magnesium was low on almost all patients. Conclusion: Even though the frequency of code blue events in solid organ transplant patients was not high, it was concerning that in many events with prior warning signs of deterioration did not result in CCRT being called. However resuscitations were prolonged reflecting the teams' investment in this patient population. This study thus highlights the importance of early involvement of CCRT in these patients.
\end{abstract}

Key words: Cardiac Arrest, Code Blue, Factors, Predictors, Solid Organ Transplant, Transplant Recipients

\section{INTRODUCTION}

Cardio Respiratory (Code Blue) arrests in solid organ transplant recipients are particularly distressing events in view of the tremendous investment by organ donors, families, and by the healthcare system. Cardiac and respiratory arrests in recipients of solid organ transplant are distressing events yet very few studies in the literature exist to help clinicians understand the incidence of such events and how to best prevent them. Toronto General Hospital is a large transplant center and is therefore uniquely situated to help improve the understanding of acute deteriorations in solid organ transplant recipients.
Transplant recipients are among the sickest hospitalised patients. Healthcare teams and caregivers are highly invested in their care and in securing the best possible outcomes post transplant. No studies have explored what early warning signs are predictive of impending cardiopulmonary arrest in solid organ transplant recipients. Moreover, no studies have explored the impact-if any-of CCRTs on preventing such events and hence in improving the quality of care of this specific and severely ill patient population. 
be selected for study.

The goal of this study was to conduct a retrospective review of all code blue events in solid organ transplant patients over a period of 5 years in order to identify unique factors that could predict such events, examine the nature and quality of actual resuscitations, and explore outcomes in this patient population. This retrospective analysis of Critical Care Response Team "no consult audit" / Code Blue data and patient chart review from 2007-2011 from the multi-organ transplant service wards and transplant step-down unit at Toronto General Hospital will explore the incidence of cardio respiratory arrests, the causes of such events and seek to identify early warning signs in order to prevent future events in this unique patient population. Thus, this study was conducted with following objectives:

- $\quad$ To identify the probable causative factors and potentially missed warning signs in the 24-hour period preceding cardio-respiratory arrests in multiorgan transplant patient populations.

- To explore outcomes including morbidity, mortality, hospital and ICU length of stay (LOS) post resuscitation in solid organ transplant recipients, and

- To improve understanding and knowledge of signs of deterioration in patient status in order to decrease morbidity, improve quality of care and survival in solid organ transplant recipients.

\section{METHODS:}

After the study was approved by University Health Network Research Ethics Board, retrospective chart review was done on all code blue events occurring in solid organ transplant patients. These Code Blue cases were identified from Critical Care Response Team (CCRT) database and the code blue resuscitation records from 2007-2011, for a period of five years. All patients who sustained cardiorespiratory arrests were included. Inclusion criteria: All patients were diagnosed to have primary angle closure in glaucoma clinic Nepal eye hospital. Age matched control group were included.

\section{Inclusion Criteria:}

- All charts of code blue calls (in solid organ transplant recipients) from the CCRT database will
- Only those patients with actual cardiac arrest and respiratory arrest will be enrolled.

- Only post solid organ transplant patients (lung, liver, kidney, and heart) will be enrolled.

\section{Exclusion Criteria:}

- False code blue calls

- Donors or patients awaiting transplants who coded.

Data was stored in a locked cabinet and was kept confidential to maintain patient's privacy. Only the investigators had access to the data and all unique patients identifying data were removed. Individual patient charts were reviewed for a period of " 24 hour prior to the arrest" to identify potential early warning signs of deterioration in clinical status. Code Blue Records were reviewed to identify resuscitation parameters that might have contributed to morbidity and mortality.

Outcome of code blue were recorded as:

- Survivor: who after the cardiac arrest had return of spontaneous circulation and was discharged from the hospital

- Non survivor: who did not have ROSC and death declared immediately after the cardiac arrest

- Delayed non survivor: If patient had ROSC but was not able to be discharged from ICU or from the hospital or if withdrawal of active treatment were undertaken

- Signs of end organ failure, transplant organ dysfunction post arrest, length of stay in hospital and in ICU, discharge data (home, rehabilitation center, chronic care) were all recorded.

Factors that could potentially have contributed to arrest events in this patient population were identified a priori by the investigators and these data points were collected from the patients' chart for the 24-48 hour period prior to the code blue arrest. In 
addition, other parameters like CCRT calling criteria, events and procedures prior to arrest, probable cause were studied. Resuscitation record was also explored to identify quality, duration and immediate event outcome. All the possible causes attributing to the arrest and the clinical status were recorded.

\section{RESULTS:}

Over the five-year period, there were sixty-three code blue calls in solid organ transplant patients out of which only $27(n=27)$ were actual code blue events with cardio respiratory arrest requiring resuscitation.

The frequency was highest in liver transplant (10), followed by lungs (8), kidney (5), double organ (kidney + liver/lung $=2$ ), and heart (1). (Figure 1)

There was one patient with Liver transplantation, who arrested twice and thus the event of Cardiac arrest was 27 total whilst the number of transplantation is only 26 patients.

Figure 1.

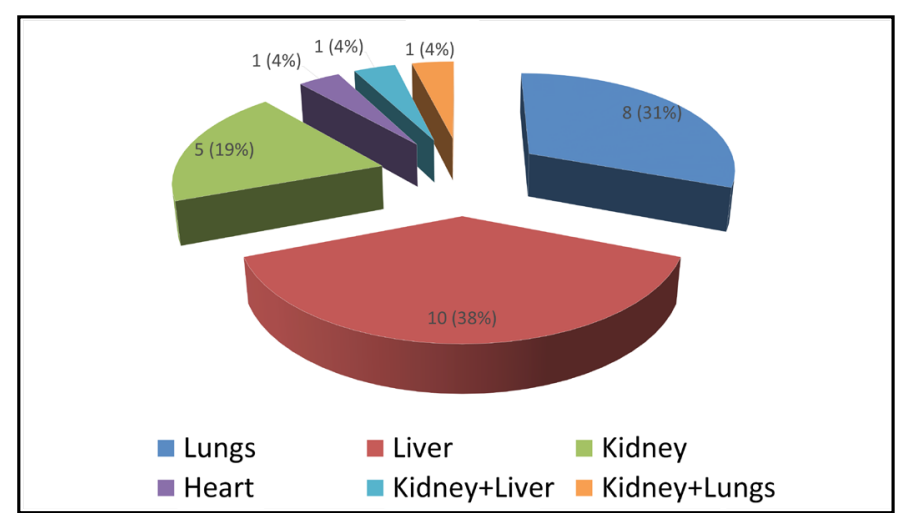

Fig:1 Frequency of arrest based on type of organ.

Signs of transplant organ dysfunction pre-code were presentinonly 2 patients $(7.40 \%)$ whereastherewasno transplant organ dysfunction in $25(92.60 \%)$ patients.

Seventeen (62.96\%) of these patients were being treated on the ward while $10(37.04 \%)$ were in the step down unit (SDU) when the arrest occurred. Transfer to a step-down unit prior to code from the floor in previous 24 hour happened in one (3.70\%) patient only while rest $26(96.30 \%)$ patients were managed in the floor itself.
Even though CCRT call criteria were met in 13 (48.14\%) of these patients, CCRT was not called. Among the CCRT Calling Criteria, Breathing criteria were met in 6 patients followed by Circulation in 5 , Neurological/Disability in 4 and Airway criteria in 2 patients only. The remaining 14 patients did not meet CCRT calling criteria and CCRT was not called.

The average 'CCRT Criteria Met time' prior to code was 24.53 hours (Average 1472.33 mins among 12 patients). (Minimum: 30 mins to Maximum: more than 72 hours).

Figure 2.

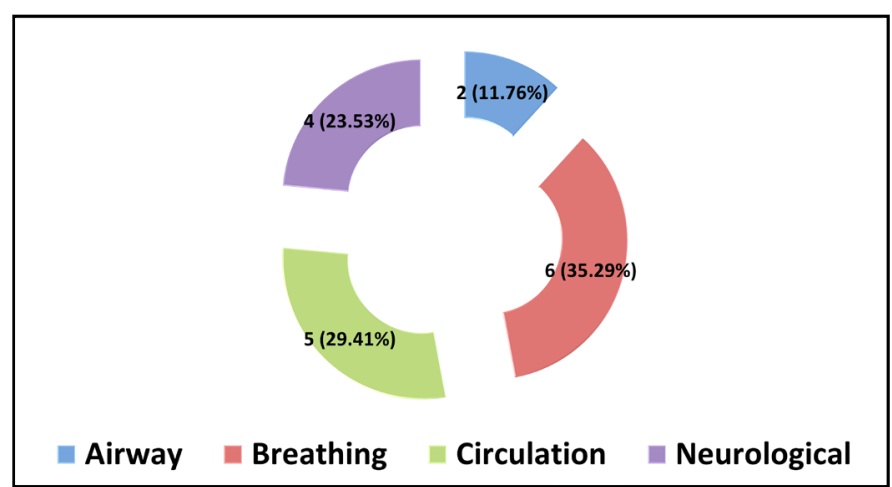

Fig:2 CCRT Calling Criteria

In most patients, 16 patients (59.25\%), a staff nurse has attended patient within $30 \mathrm{~min}$ prior to code blue. However, the average time interval being attended prior to code blue was $115.81 \mathrm{mins}$ ( 5 to 600 mins) for nurses and 12.77 hours ( 0.5 to 24 hours) for physicians.

All patients had been treated in the ICU prior to code blue event and the time interval from ICU discharge to code blue call averaged 5.44 days (1-40 days).

Some common factors that were present in these code blue patients were documented source of infection $(11,40.74 \%)$, blood transfusion $(7,23.9 \%)$, surgical event as cause of arrest $(6,22.22 \%)$, procedures within 24 hours $(6,22.22 \%)$ and dialysis within 24 hours $(5,18.51 \%)$.

Considering infection as a major factor of code blue arrest, the frequency of infection in patients who had Code Blue was in 11 (40.74\%) patients only. The common infections were Lung infection in three patients (as evidenced by BAL, Aspergillous 1, Nocardia -1, Aspergillous + MDR Pseudomonas - 1); Blood culture was positive in three patients 
(CMV - 2, Pseudomonas - 1); both Lung Infection \& Blood culture positive in two patients (Nocardia + Candida, Aspergillous + CNST), Intrabdominal infection in two patients (Enterococcus faecium); Urine (Enterococcus) and wound infection (Serratia marcescens) in one patient and Spinal abscess (Staph aureus) in one patient.

Analyzing surgical events or cause of code blue, bleeding occurred in three patients (11.11\%), intra-abdominal abscess in one $(3.70 \%)$, massive hemoptysis in one (3.70\%) and bleeding after paracentesis occurred in one (3.70\%) patient.

Other event that could have contributed to arrest was aspiration, was noted during CPR/Intubation in four (14.81\%) patients.

Another possible preventable cause of arrest occurred in one patient who had Opioid overdose immediate post liver transplant and had respiratory depression and arrested.

It was also found that of all the electrolytes and acid base parameters, only serum magnesium was low on almost all patients who arrested but its significance in survivors was not tested.

At the call of Code Blue, the time for arrival of Code Blue Team was mentioned in only in five patients record and the average time for arrival of Code Blue Team was: 3.4 mins

Considering time to intubation, the average duration from Code Blue call time to intubation time was 9.42 mins ( 2 mins to 35 mins). Out of 27 patients, 22 patients required respiratory support during Code Blue and one patient had tracheostomy and only 21 patients out of 26 had the time for intubation mentioned.

At the onset of the code blue, 24 patients had nonshockable rhythm (Asystole - 10, PEA - 6, respiratory arrest with pulse - 8) while three had shock-able rhythm. ROSC was seen in a total of 17 patients (62.97\%); in whom five had Asystole, five had PEA and seven had NSR at onset. No patients with an initial shockable rhythm survived.

Average duration of resuscitation was 43.13 mins $(11-113$ mins) while time to ROSC in survivors was 10.33 mins.
Nine patients who had initial ROSC later died; five after life support was withdrawn due to poor neurological outcomes. Such withdrawals of life support occurred in an average after 11 days (2-21 days).

In these patients after ROSC, Therapeutic Hypothermia was induced and maintained in only one patient. ( 1 in $17,5.88 \%$ )

After the Code Blue arrest, these patients were admitted in ICU (LOS ICU) for 14.35 days (2 days to 48 days) and in Hospital for 52.7 days (2 days to 292 days) in an average.

Out of the eight survivors, only three were discharged home, three were transferred to another hospital, one was discharged to chronic care facility, and one was discharged to rehabilitation facility.

Figure 3.

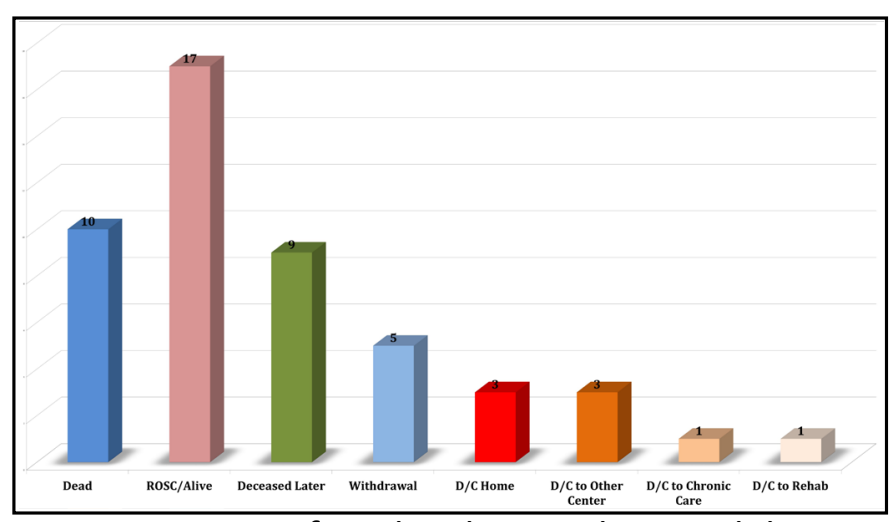

Fig:3 Outcome of Code Blue in these solid organ transplant patients.

\section{DISCUSSION:}

Solid organ transplantation has revolutionized the lives of organ and there have been improvements in both patient and allograft survival rates through advances in medical therapies and surgical techniques. However, significant ongoing morbidities are still associated with the patients' underlying chronic conditions and transplantation. ${ }^{1}$

Transplant recipients are among the sickest hospitalised patients. Healthcare teams and caregivers are highly invested in their care and in securing the best possible post transplant outcomes. No studies have explored what early warning signs are predictive of impending cardiopulmonary arrest in solid organ transplant recipients. Moreover, no 
studies have explored the impact-if any-of CCRTs on preventing such events and hence in improving the quality of care of this specific and severely ill patient population.

Numerous studies in the literature have shown the efficacy of Critical Care Response Teams (CCRT) in preventing cardiac arrests in general hospital patients in various ways. Bellomo et $\mathrm{al}^{2}$ showed that implementation of Medical Emergency Team (MET) reduced cardiac arrest by $65 \%$, death from cardiac arrest by $56 \%$ and duration of post arrest by $80 \%$, and in-patient deaths by $25 \%$. Another study by Sharek PJ et $\mathrm{al}^{3}$ showed that implementation of RRT was associated with a statistically significant reduction in hospital wide monthly mortality rate by $18 \%$ and code rate outside of the pediatric ICU by $71 \%$. According to a systematic review by Paul S. Chan et $\mathrm{al}^{4}$, RRT implementation in adults was associated with $33.3 \%$ reduction in the rates of cardiopulmonary arrest outside the ICU but was not associated with lower hospital mortality rates. Moreover, RRT activation resulted in early patient transfer to a higher level of care and avoided progression to cardiac arrest.2 The introduction of a MET was associated with reduction of total hospital death and reduction of preventable cardiac arrest and death with increased survival in wards of a paediatric hospital. MET calling criteria identified some but not all children at risk of unexpected cardiac arrest and death. ${ }^{5}$

Our study also showed that there were many incidences when CCRT calling criteria was met but CCRT was not called or involved late. This also leads to delayed patient management and adverse outcomes. Marshall et al and Lindy et al and Jane Saucedo Braaten have extensively elaborated the barriers to CCRT calls and related factors and their solutions. ${ }^{6,7,8}$

Considering causes of death in solid organ transplant patients, Sanromán Budiño et al, studied 156 autopsies including 76 heart, 32 liver, 29 kidney, and 19 lung transplant recipients, and found that infections were the most common cause of death in all groups, varying from $21 \%$ in heart to $63 \%$ in lung recipients.9 Acute rejection, chronic rejection, and malignancies only appeared as the cause of death in heart recipients $(14.5 \%, 9.2 \%$, and $4 \%$, respectively. In all groups there were a significant percentage (about 30\%) of other pathologies that were responsible for death, such as pulmonary embolism, central nervous system pathology, acute pancreatitis, digestive hemorrhage, and acute myocardial infarction. The results emphasize that infections were the main cause of death within the first year post transplant, independent of the organ transplanted.

As per review by Briggs Douglas J, and another study by Neale et al, the common causes of death in post kidney transplant patients were cardiovascular, malignancy and infections. 10,11 The most common cause of death in lung transplantation was infection followed by bronchiolitis obliterans. 12

Cardiac complications after Orthoptic Liver Transplantation are common and were the leading cause of death. Adverse intraoperative cardiovascular events, previous cardiac disease, and advanced liver disease as quantified by i-MELD score predicted postoperative cardiac complications and death in Liver Transplant recipients.13

According to The Registry of the International Society for Heart and Lung Transplantation, the most common identifiable causes of death in the first 30 days post-transplant were graft failure (lung or heart) and technical complications. Of deaths within 30 days, those transplanted for PAH had a higher percentage of deaths due to non-CMV infections. After the first year, OB/BOS/late graft failure (lung or heart) and non-CMV infections were the most common causes of mortality. Cardiovascular causes of death accounted for a smaller, but important, proportion of the deaths. 14

But none of the studies pointed to immediate causes of ICU admission and death in solid organ transplant patients and elaborated about causes of death.

\section{CONCLUSION:}

The frequency of code blue events was not high in solid organ transplant patients but concerningly, in view of such massive investments in these patient populations, in many events with prior warning signs of deterioration did not result in CCRT being called. Most events occurred beyond the normal CCRT follow up period indicating perhaps a need for more prolonged follow up or more vigilance 
on part of transplants teams. Resuscitations were prolonged reflecting the teams' investment in this patient population. In all of the patients, the graft function was maintained.

\section{ACKNOWLEDGEMENTS:}

The authors highly acknowledge all the Critical Care Response Team and Critical Care Nurses at the Toronto General Hospital, University Health Network for their dedication toward the CCRT and their support during the study.

\section{REFERENCES:}

1. Kim JJ et al. Solid organ transplantation in children. Clinics. 2014;69(S1):28-38.

2. Bellomo R, Goldsmith D, Uchino S, Buckmaster J, Hart G, Opdam H, et al. Prospective controlled trial of effect of medical emergency team on postoperative morbidity and mortality rates. Crit Care Med. 2004;32:916-21.

3. Sharek PJ, Parast LM, Leong K, Coombs J, Earnest $K$, Sullivan J, et al. Effect of a rapid response team on hospital wide mortality and code rates outside the ICU in a Children's Hospital. JAMA. 2007;298:2267-74.

4. Paul S. Chan, Renuka Jain, Brahmajee K. Nallmothu, Robert A. Berg, Comilla Sasson, Rapid Response Teams: A Systematic Review and Metaanalysis Arch Intern Med. 2010;170(1):18-26.

5. Offner, Patrick J, Heit, Joseph; Roberts, Robin. Implementation of a Rapid Response Team Decreases Cardiac Arrest Outside of the Intensive Care Unit. Journal of Trauma-Injury Infection \& Critical Care. 2007;62(5):1223-1228. doi: 10.1097/TA.0b013e31804d4968

6. Marshall et al. Why don't hospital staff activate the rapid response system (RRS)? How frequently is it needed and can the process be improved? Implementation Science. 2011;6:39.

7. King $L$, et al. Are there still barriers to MET callsMetropolitan and regional nurses' and midwives' perspectives? Collegian (2018).

8. Jane Saucedo Braaten. Hospital System Barriers to Rapid Response Team Activation: A Cognitive Work Analysis. AJN. 2015;115:2-4.
9. Sanromán Budiño B1, Vázquez Martul E, Pértega Díaz S, Veiga Barreiro A, Carro Rey E, Mosquera Reboredo J. Autopsy-determined causes of death in solid organ transplant recipients. Transplant Proc. 2004;36(3):787-9. 